\title{
Évaluation du métabolisme énergétique de la boxe anglaise de haut niveau de performance
}

\author{
Jean-Marc Vallier, Jeanick Brisswalter, Christine Hanon
}

Institut National du Sport et de l'Education Physique, 11, avenue du Tremblay, 75012 Paris, France

Article paru dans : Science \& Sports, 1995, volume 10, numéro 3, pp. 159-162

La boxe anglaise est un sport technique occasionnant une dépense énergétique importante (Dal Monte et Menchinelli, 1986 ; Victorov et al, 1976). L'entraîneur doit intégrer les deux composantes, technique et énergétique, dans la préparation des boxeurs. L'entraînement technique se fait lors de séances spécifiques de type « frappe au sac » ou combat. L'entraînement physique se fait soit sous forme de course à pied ou de « saut à la corde», soit lors de séances plus spécifiques comme la «frappe au sac». Ces séances d'entraînement font partie de la " culture pugilistique » et ont été peu modifiées ces dernières années.

Aussi, pour définir la programmation de l'entraînement, est-il important de connaître la nature des sollicitations du métabolisme énergétique lors de ces séances. Or peu d'études dans le domaine de la boxe anglaise ont été réalisées. Ces études ont utilisé la fréquence cardiaque. Victorov et al (1976) ont utilisé la fréquence cardiaque pour évaluer quantitativement l'intensité des charges d'entraînement.

Dal Monte et Menchinelli (1986) ont étudié l'évolution de la fréquence cardiaque au cours de combats amateurs de boxe anglaise. Leur conclusion est que les combats sollicitent alternativement les métabolismes aérobie et anaérobie. Le fait que ces études utilisent uniquement la fréquence cardiaque ne permet pas de déterminer avec précision la filière énergétique dominante pour chaque exercice.

C'est pour cette raison que nous avons essayé de mettre en relation dans cette étude la lactatémie et la fréquence cardiaque mesurées sur le terrain et les mêmes paramètres mesurés en laboratoire pour étudier le métabolisme énergétique sollicité lors des séances d'entraînement chez des boxeurs de haut niveau.

\section{MATÉRIELS ET MÉTHODES}

\section{Les sujets}

Il s'agit de 16 boxeurs amateurs français de niveau national ou international qui s'entraînent deux fois par jour. Leur âge est de 20,7 $\pm 1,9$ ans, leur taille est de $176 \pm 9 \mathrm{~cm}$, leur poids est de $68,4 \pm 10,0 \mathrm{~kg}$.

\section{Évaluation en laboratoire}

Une épreuve d'effort de détermination de la consommation maximale d'oxygène (VO2 max) a été réalisée au laboratoire d'exploration fonctionnelle de l'Insep selon la méthode décrite par Joussellin et Legros (1990). Le sujet court sur un tapis roulant avec une pente de 3\%. L'effort est discontinu, chaque palier d'effort de 4 minutes est entrecoupé d'un repos d'une minute. La vitesse de départ est de $8 \mathrm{~km} . \mathrm{h}^{-1}$, l'incrémentation de la vitesse est de $2 \mathrm{~km} \cdot \mathrm{h}^{-1}$ à chaque palier. La vitesse du tapis est vérifiée de façon continue pendant le test par une cellule photoélectrique. Les gaz expirés sont recueillis pendant les 2 dernières minutes de chaque palier par un analyseur en circuit ouvert de type CPX Medicalgraphics. La fréquence cardiaque est enregistrée en continu sur un scope. Un prélèvement sanguin est effectué au lobe de l'oreille entre chaque palier pour mesurer la lactatémie par microdosage sur Microzym-L (SGI). La zone de transition aéro-anaérobie est estimée pour une lactatémie de 4 mmol.1 ${ }^{-1}$ (Mader et al, 1976). Le test est arrêté lorsque $\mathrm{VO} 2$ atteint un plateau ou bien lorsque la fréquence cardiaque est proche de la fréquence cardiaque maximale théorique avec un quotient respiratoire supérieur à 1,1 (Vandewalle et Friemel, 
1989).

Sur le terrain, les paramètres mesurés sont la fréquence cardiaque et la lactatémie. La fréquence cardiaque est enregistrée de façon continue par un Baumann BHL 6000. Pour chaque série ou chaque reprise, la fréquence cardiaque est exprimée sous la forme d'un pourcentage de la fréquence cardiaque maximale mesurée lors du test d'effort en laboratoire (Karvonen et Vuorimaa, 1988).

Un prélèvement sanguin effectué au lobe de l'oreille au repos et à la fin de chaque série ou reprise permet de mesurer la lactatémie.

Les séances d'entraînement sont de deux types : « frappe au sac » et " saut à la corde ». Elles sont effectuées suivant le même schéma de trois séries de 3 minutes entrecoupées d'une minute de récupération. L'intensité de l'exercice qui doit être fait à vitesse gestuelle maximale est contrôlée verbalement par l'entraîneur.

Des combats ont été organisés entre les boxeurs de l'Insep et ceux de l'école interarmées des sports. Ces combats ont été réalisés en trois reprises de 3 minutes entrecoupées d'une minute de récupération, leur intensité a été comparable à une compétition car il existait une rivalité entre ces combattants de niveau national.

\section{Analyse statistique}

La moyenne et l'écart type de chaque paramètre ont été calculés.

L'évolution des paramètres physiologiques lors des séries d'une même séance ainsi que la comparaison des différents types d'exercice sont analysées à partir d'une étude de variance à deux facteurs (effet du type d'exercice, effet de répétition des mesures). Le seuil de signification est fixé à $p<0,05$.

Enfin, le test post-hoc de Dunnet a permis de comparer chaque mesure avec la mesure effectuée lors de la série précédente. Le seuil de signification est fixé à $p<0,05$.

\section{RÉSULTATS}

Les résultats du test d'effort de détermination de la VO2 max sont présentés dans le tableau 1.

La figure 1 représente l'évolution de la lactatémie lors des deux types d'entrainement et lors du combat. Il existe un effet de répétition des mesures uniquement lors du combat. Cela est vérifié par le test post-hoc de Dunnet qui montre une augmentation statistiquement significative de la lactatémie aux deuxième et troisième reprises. Lors des deux types d'entraînement (« saut à la corde » et « frappe au sac »), la lactatémie est statistiquement stable. Il n'existe pas d'effet du type d'exercice entre la frappe au sac et le saut à la corde. En revanche, il existe un effet type d'exercice entre le combat et les deux types d'entraînement.

La figure 2 représente l'évolution de la fréquence cardiaque (FC) lors des deux types d'entraînement et lors du combat. Le « saut à la corde » est la séance entraînant la moindre élévation de la FC. Les valeurs de FC mesurées se situent entre $85 \%$ et $89 \%$ de la FC maximale obtenue en laboratoire. Lors de la « frappe au sac », la FC est plus élevée : entre $88 \%$ et $94 \%$ de la FC maximale. L'augmentation de la lactatémie lors des deuxième et troisième séries est statistiquement significative par le test post-hoc de Dunnet. Enfin, le combat est la séance qui augmente le plus le rythme cardiaque (la FC atteint la FC maximale à la fin du combat). L'augmentation de la FC n'est cependant significative qu'à la deuxième série.

\section{DISCUSSION}

Le test d'effort en laboratoire montre que la consommation maximale d'oxygène (VO2 max) des boxeurs est de 62,2 $\pm 3,1$ ml.min-1... $\mathrm{kg}^{-1} 1$

Cette valeur, importante pour des sportifs non spécialistes de course à pied, témoigne d'un potentiel aérobie important. Ce résultat est identique à celui trouvé par Joussellin et Legros (1990) chez les boxeurs de niveau national. Les valeurs de lactatémie et de fréquence cardiaque atteintes lors du palier maximal sont relativement peu élevées. Enfin, la zone de transition aéro-anaérobie déterminée pour une lactatémie de $4 \mathrm{mmol}^{-1} \mathrm{l}^{-1}$, est assez proche du palier maximal. Cela indique que les boxeurs ont développé par leur entraînement une endurance aérobie importante (Kinderman et al, 1979). Ces trois critères, VO2 max relativement élevé, zone de transition aéro-anaérobie élevée, lactatémie et fréquence cardiaque maximales basses, indiquent que les boxeurs ont un métabolisme aérobie très développé.

Les mesures de lactatémie et de fréquence cardiaque effectuées sur le terrain nous ont permis d'estimer 
la dépense énergétique des entrainements et des combats. Ces séances diffèrent de par les groupes musculaires qu'elles mettent en jeu. Le " saut à la corde " et le test sur tapis roulant mobilisent essentiellement les membres inférieurs. La « frappe au sac » mobilise essentiellement les membres supérieurs. Le combat sollicite à la fois les membres supérieurs et inférieurs. Or, VO2 max et production $\mathrm{d}$ ' acide lactique sont proportionnelles à la masse musculaire mise en jeu lors des mesures (Se- cher et $a l, 1974)$. L'utilisation de la relation linéaire FC-VO2 établie lors du test sur tapis roulant ne permet pas de quantifier la dépense énergétique d'un exercice mobilisant des groupes musculaires différents à partir d'une valeur de fréquence cardiaque enregistrée sur le terrain. L'étude de l'évolution de la lactatémie (stabilité ou non au cours des différentes reprises) pour déterminer la filière métabolique prépondérante lors d'un exercice de terrain semble moins dépendante de la masse musculaire mise en jeu (Di Prampero, 1981).

La dépense énergétique des boxeurs a été évaluée lors de combats dont l'intensité était proche du rythme de compétition. L'évolution de la fréquence cardiaque et de la lactatémie témoigne de l'intensité importante des combats. La fréquence cardiaque est croissante tout au long des combats pour atteindre la valeur maximale mesurée lors du test d'effort en laboratoire. De même, la lactatémie augmente de façon significative à chaque reprise.

Cette absence de stabilité montre que la demande énergétique au cours d'un combat de boxe dépasse les possibilités de la filière aérobie (Di Prampero, 1981) et sollicite la glycolyse anaérobie. Un combat de boxe peut donc être considéré comme un exercice anaérobie intermittent, ce qui est proche de la notion d'activité alternativement aérobie et anaérobie proposée par Dal Monte et Menchelli (1986).

Les séances de préparation physique réalisées dans la salle de boxe ont été évaluées à partir de la fréquence cardiaque et de la lactatémie. La séance de « saut à la corde » s'est révélée comme étant une séance peu intensive. La fréquence cardiaque ne dépasse pas $89 \%$ de la fréquence maximale enregistrée lors du test de laboratoire. Par ailleurs, la lactatémie et la fréquence cardiaque sont statistiquement stables lors des trois séries. Ce type d'entraînement sollicite donc préférentiellement le métabolisme aérobie.

La séance de « frappe au sac » est un peu plus intensive (la fréquence cardiaque atteint $94 \%$ de la fréquence maximale) mais elle reste essentiellement aérobie. En effet, la lactatémie est statistiquement stable tout au long des trois séries de 3 minutes. La comparaison des résultats obtenus lors des combats, d'une part, et lors des séances de préparation physique, d'autre part, montre qu'il y a une inéquation entre les caractéristiques de la sollicitation énergétique utilisée par les boxeurs dans la compétition et celles développées par l'entraînement. Plusieurs hypothèses peuvent être proposées pour expliquer ce décalage. Tout d'abord, le « saut à la corde » fait partie de la « culture pugilistique ». Par conséquent, les boxeurs de haut niveau ont développé une économie gestuelle dans cette pratique et semblent principalement solliciter la filière aérobie pour la réaliser. Ensuite, la « frappe au sac » se pratique de façon statique devant le sac, seul le haut du corps travaille. Les groupes musculaires sollicités ne paraissent pas assez importants pour déclencher la filière anaérobie.

Enfin, l'entraînement en course à pied, qui n'a pas été évalué dans cette étude, est pratiqué par chaque boxeur sous forme de « footing » libre. Cet exercice doit par conséquent être essentiellement aérobie.

En conclusion, toutes les séances de préparation physique des boxeurs sollicitent essentiellement le métabolisme aérobie.

Cela permet d'expliquer leur potentiel aérobie important révélé lors du test d'effort sur tapis roulant. En revanche, cette préparation physique ne répond qu'imparfaitement à la demande énergétique des combats qui sollicitent nettement le métabolisme anaérobie.

Afin d'améliorer leurs performances au cours des combats, les boxeurs devraient mieux orienter leur préparation physique dans le sens d'un développement de la puissance anaérobie.

\section{RÉFÉRENCES}

Dal Monte A, Menchinelli C. Aspetti fisiologici del pugilato. Med Sport 1986;39:103-11

Di Prampero PE. Energetics of muscular exercise. Rev Physiol Biochem Pharmacol 1981;89:143222

Joussellin E, Legros P. Exploration du métabolisme énergétique chez le sportif de haut niveau. Paris : Insep publications, 1990 
Karvonen J, Vuorimaa T. Heart rate and exercise intensity during sports activities. Pract Appl Sports Med 1988;5:303-12

Kinderman W, Simon G, Keul J. The significance of the aerobic-anaerobic transition for the deternination of work load during intense training. Eur J Appl Physiol 1979;42:25-34

Made A, Liesen H, Heck H, Philippi H, Schurch PM, Hollmann W. Zur Beurteilung der sportartspezifischen Ausdauerleistungsfahigkeit. Sportarzt Sportmed 1976;27:80-88, 109-12

Secher NH, Ruberg-Larsen N, Binkhorst RA, Bonde-Petersen F. Maximal oxygen uptake during arts crancking and combined arm plus leg exercise. J Appl Physiol 1974;36:515-8

Vandewalle H, Friemel F. Tests d'évaluation de la puissance maximale des métabolismes aérobie et anaérobie. Science \& Sports 1989;4:265-79

Victorov IB, Nikiforov B, Tcherniak AV. Évaluation quantitative de l'intensité des charges d'entraînement en boxe. Teoria i praktika fizitcheskoj kultury 1976;11:20-3 (traduction Insep) 
Tableau 1. Caractéristiques des paramètres physiologiques mesurés lors de l'épreuve maximale sur tapis roulant $(\mathrm{n}=16)$.

\begin{tabular}{|c|c|}
\hline Paramètres & Moyenne \pm écart type \\
\hline VO2 max (ml.min $\left.{ }^{-1} \cdot \mathrm{kg}^{-1}\right)$ & $62,2 \pm 3,1$ \\
\hline Vitesse de course à VO2 $\max \left(\mathrm{km} \cdot \mathrm{h}^{-1}\right)$ & $17 \pm 1,8$ \\
\hline Lactatémie à VO2 max (mmol. $\left.1^{-1}\right)$ & $8,7 \pm 2,2$ \\
\hline Fréquence cardiaque à V02 max (bpm) & $192,2 \pm 7,5$ \\
\hline $\begin{array}{l}\left.\text { Vitesse de course (km.h- }{ }^{1}\right) \text { pour une } \\
\text { lactatémie de } 4 \text { mmol.1- }{ }^{1}\end{array}$ & $14,6 \pm 1,2$ \\
\hline $\begin{array}{l}\text { Fréquence cardiaque (bpm) pour une pour une } \\
\text { lactatémie de } 4 \text { mmol.1- }{ }^{1}\end{array}$ & $181,3 \pm 7,3$ \\
\hline $\begin{array}{l}\text { Pourcentage de VO2 max pour une } \\
\text { lactatémie de } 4 \text { mmol.1- }{ }^{1}\end{array}$ & $89,1 \pm 4,4$ \\
\hline
\end{tabular}

Fig 1. Évolution de la lactatémie (moyenne $\pm d s$ ) au cours des séries en fonction du type d'entrainement. * Les valeurs de lactatémie présentent une augmentation significative avec la série précédente pour $\mathrm{p}<$ 0,05 .

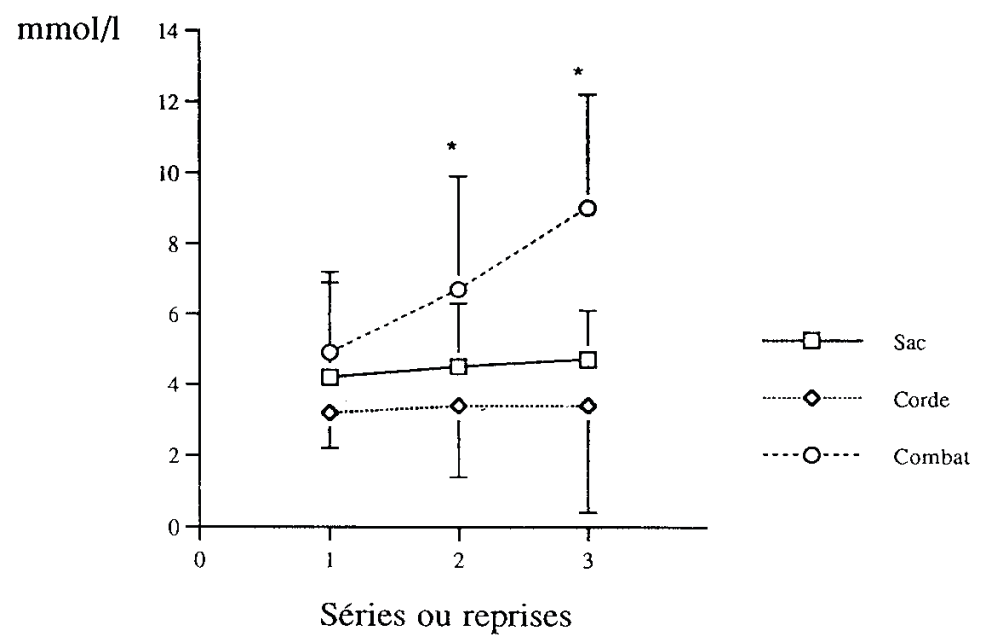


Fig 2. Évolution de la fréquence cardiaque (moyenne $\pm d s$ ) au cours des séries en fonction du type d'entraînement. * Les valeurs de fréquence cardiaque présentent une augmentation significative avec la série précédente pour $\boldsymbol{p}<0,05$.

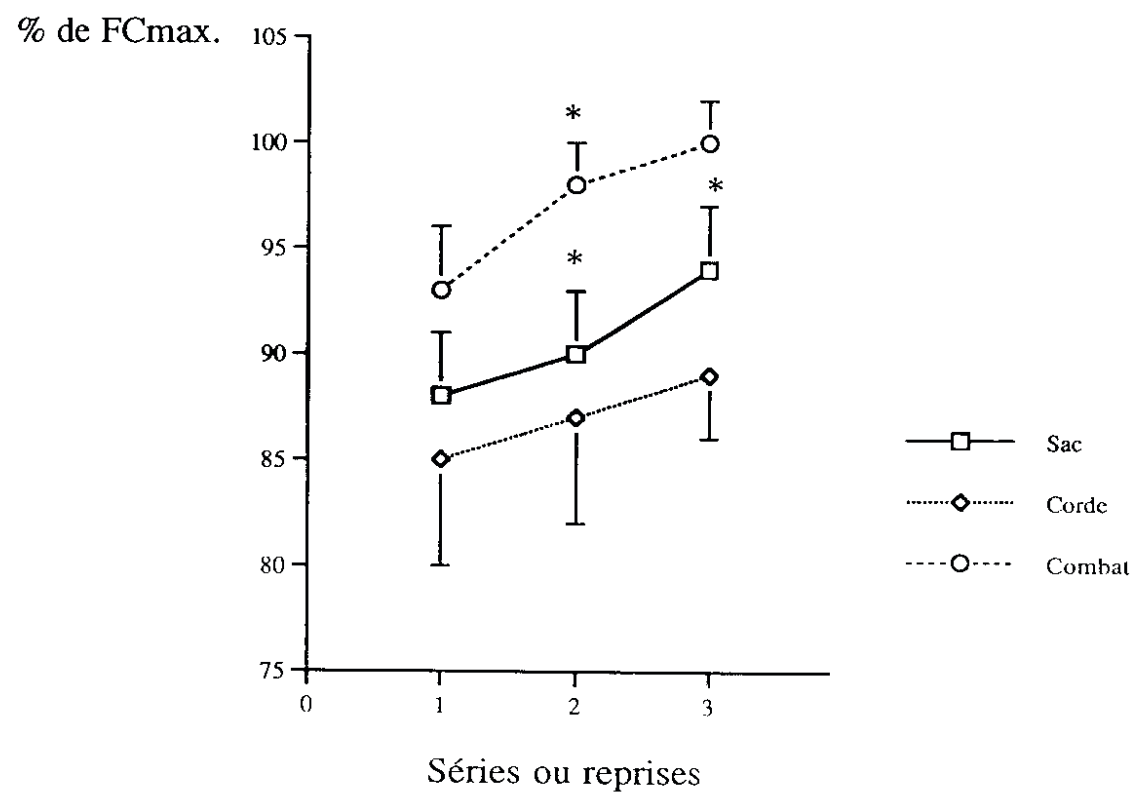

